\title{
Signatures of heating processes in the Galactic thin disk
}

\author{
Birgitta Nordström \\ The Niels Bohr Institute, University of Copenhagen \\ Juliane Maries Vej 30, DK-2100 Copenhagen, Denmark \\ E-mail: birgitta@astro.ku.dk
}

\begin{abstract}
The term "heating" is used loosely to refer to a range of processes that result in an increase in velocity dispersion with age for subgroups of disk stars. We briefly summarise the observational basis for studies of disk heating and show that qualitative differences exist between the evolution of the in-plane and vertical motions. Ways to discriminate between various heating scenarios are discussed; the most recent galaxy merger simulations may in fact suggest that discrimination on purely kinematic grounds might be unfeasible, even with large samples of stars with excellent ages.
\end{abstract}

Keywords. stars: kinematics, Galaxy: solar neighborhood, Galaxy: disk, Galaxy: evolution, galaxies: kinematics and dynamics

\section{Introduction}

Observations demonstrate that old stellar populations in the Galactic disk show larger random motions (velocity dispersions) than groups of younger stars; see Wielen (1977) for a classic reference. This observation may reflect ( $i)$ : initial conditions in a monolithic collapse scenario for the disk; (ii): gradual scattering of the initially circular orbits of newborn stars by spiral arms or massive objects within the disk itself, such as giant molecular clouds (GMCs) or ; (iii): the effect of the gradual accretion of dwarf galaxies into the disk in a hierarchical merger scenario. Thus, the velocities (and, by inference, Galactic orbits) of disk stars contain vital information on the formation and evolution of the disk.

The term "disk heating" is often applied loosely to the sum of the effects that may cause larger velocity dispersions in old stars. Strictly speaking, however, "heating" should only be used to refer to processes that, over time, inject kinetic energy into the random component of the stellar motions. Fossilised initial conditions for, e.g., halo, perhaps thick disk stars are not the result of "heating", nor is the combination of ordered motions of subgroups of stars such as moving groups or differential effects of Galactic rotation. Disentangling these various effects from the observations and placing them in proper historical perspective is not a simple matter, however.

In order to understand the origin of the present assemblage of disk stars, it is therefore necessary to quantify the kinematic properties of subpopulations in the disk and characterise the origin of their stars as accurately as possible. This contribution provides a brief review of the observational material available for such studies and what can be learned from the data at present.

\section{Available data sets}

The obvious requirement for detailed studies of the kinematic evolution of disk stars with time is a set of complete kinematic and astrophysical data for a large sample of stars: 
Even after subdividing the stars by such parameters as age, metallicity, or type of Galactic orbit, the resulting subsamples must be large enough to reduce the sampling noise in the resulting velocity dispersions to a level where observed differences are statistically significant. E.g., the often-quoted paper by Quillen \& Garnett (2001) was based on less than 200 stars - clearly insufficient for their conclusions to be robust. Freedom from kinematic selection biases is an equally important condition.

The Hipparcos mission yielded large numbers of stars with accurate parallaxes, proper motions, and hence tangential velocities. A kinematically unbiased subsample of $\sim 12,000$ main-sequence stars was analysed in considerable detail by Dehnen \& Binney (1998). However, radial velocities, and thus true three-dimensional velocity vectors, were missing for most stars, and the $B-V$ colour index was used as a crude proxy for age. The lack of multiple radial velocities also precluded the detection of close (spectroscopic) binaries in the sample.

The lack of radial velocities was mitigated in part by Famaey et al. (2005), who provided accurate radial velocities for $\sim 6,000$ northern $\mathrm{K}$ and $\mathrm{M}$ giants observed by Hipparcos. They computed the full Galactic velocity components $U, V, W$ and analysed the systematic patterns in the data with sophisticated maximum likelihood techniques. Rough ages were estimated from isochrones for the subgroupings they defined. The strength of this data set is the large sample of accurate velocities and freedom from kinematic bias; the weakness is the lack of full sky coverage and of reliable age estimates for the individual stars.

Most recently, the RAVE project (Zwitter et al. 2008) has started producing radial velocities of large numbers of faint southern stars, selected without kinematic bias. Again, the lack of complete sky coverage and accurate stellar parameters for most stars, including accurate parallaxes, proper motions, and accurate ages, impedes the use of these data for the purposes discussed here.
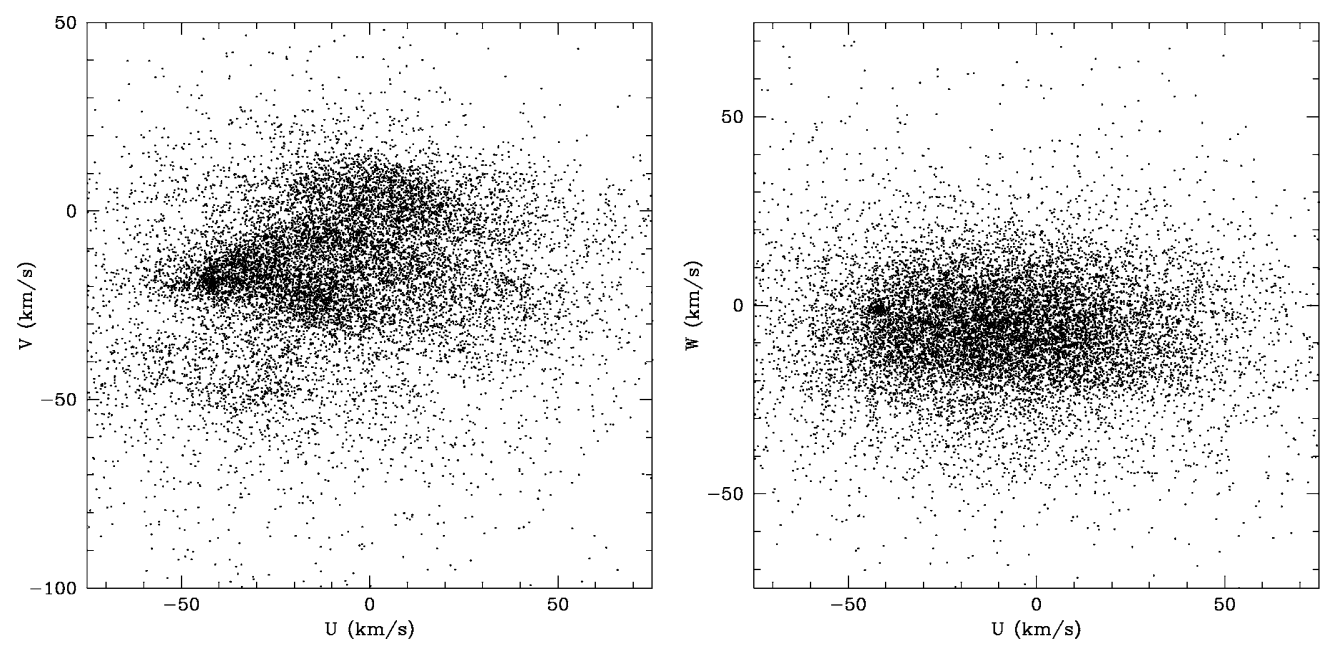

Figure 1. The observed $U V$ (left) and $U W$ planes (right) for the GCS stars. $\mathrm{W}$ is the velocity perpendicular to the Galactic plane.

The currently largest and, by design, most complete data set from which disk heating can be studied is the Geneva-Copenhagen Survey of the Solar Neighbourhood by Nordström et al. (2004; GCS I). The GCS contains a magnitude-limited, kinematically unbiased sample of $\sim 14,000 \mathrm{~F}$ and $\mathrm{G}$ dwarf stars with Hipparcos parallaxes and Tycho-2 proper motions, accurate, multiple radial-velocity observations (important for 
binary detection), and derived $U, V, W$ velocity components and Galactic orbital parameters. Metallicities and other astrophysical parameters were determined from Strömgren uvby $\beta$ photometry, and ages and their uncertainties were computed by the method of Jørgensen \& Lindegren (2005). The key calibrations were revisited and updated by Holmberg et al. (2007; GCS II), and the revised Hipparcos parallaxes by van Leeuwen (2007) in Holmberg et al. (2009; GCS III), which contains the latest version of the data set. Most stars are within $150 \mathrm{pc}$ of the Sun, and the sample is essentially volume complete out to $\sim 40 \mathrm{pc}$.

In the following, we illustrate the use of the data in studies of the kinematic history of the Galactic disk. There are qualitative differences between the in-plane velocities and those perpendicular to the plane, so the two cases will be discussed separately.

\section{Motions in the Galactic plane}

The qualitative difference between the in-plane and vertical velocity distributions is obvious from Fig. 1. The $U V$ plane shows anything but the sum of smooth, Gaussian distributions that would correspond to a simple model of thin and thick disk plus a sprinkling of halo stars. Apart from the Hyades (prominent at left), the stars are distributed in a number of bands that must correspond to distinct dynamical features such as spiral arms, moving groups, or other phenomena. These bands are not visible in the $U W$ plane, however, indicating that different mechanisms dominate the in-plane and vertical dynamical histories.

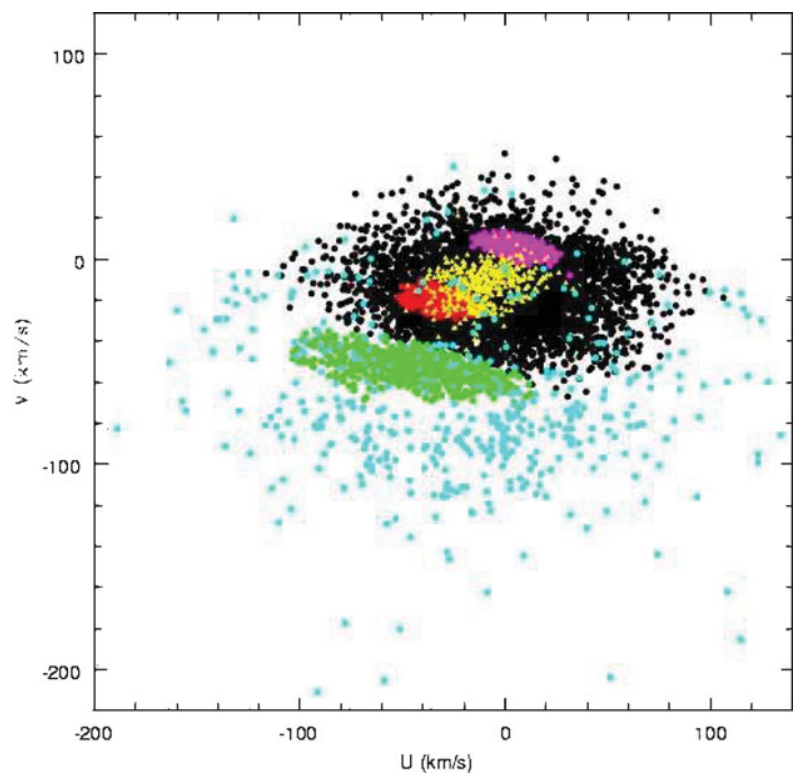

Figure 2. The kinematic decomposition by Famaey et al. (2005) of the $U V$ plane for their stars. The smooth background of black and outer blue points shows the general thin and thick disk (plus halo) population. The central (yellow) group consists of the youngest stars, with the Sirius (magenta) and Hyades-Pleiades (red) groups directly above and below. The dense band of green points below the main disk concentration is the Hercules stream.

The in-plane velocity distribution was analysed in detail by Famaey et al. (2005), who used a maximum likelihood technique to identify a number of distinct dynamical groups 
in their $U V$ plane; see Fig. 2. Already their limited age and metallicity data enabled them to conclude that these groups we not dominated by stars of a common origin, i.e. with the same age and chemical composition, a conclusion that has been refined later with better data, e.g. high resolution spectroscopic data for the Hercules stream by Bensby et al. (2007).

Thus, these features appear to arise from some mechanism(s) in the disk capable of dynamically focusing stars into similar orbits. E.g., the Hercules stream has been ascribed to the Outer Lindblad Resonance with the Galactic bar (Dehnen 2000, Fux 2001), but other mechanisms are possible, such as stationary or transient spiral arms. As pointed out by Seabroke \& Gilmore (2007), a simple velocity dispersion is then inadequate to characterise the dynamical state of disk stars of a given age, and more detailed dynamical simulations are needed to clarify the origin of the observed in-plane motions. "Disk heating" then becomes an integral chapter of the origin and evolution of the Milky Way Galaxy itself.

\section{The vertical kinematics}

As suggested by Fig. 1 (right), the vertical $(W)$ motions of disk stars do not reflect the complicated substructure seen in the $U V$ plane. Instead, as noted by Seabroke \& Gilmore (2007), the $W$ velocities show an essentially pure Gaussian distribution at all ages (see Fig. 3), suggesting that "heating" is indeed an appropriate term for the gradual increase of random motions seen in the vertical direction.
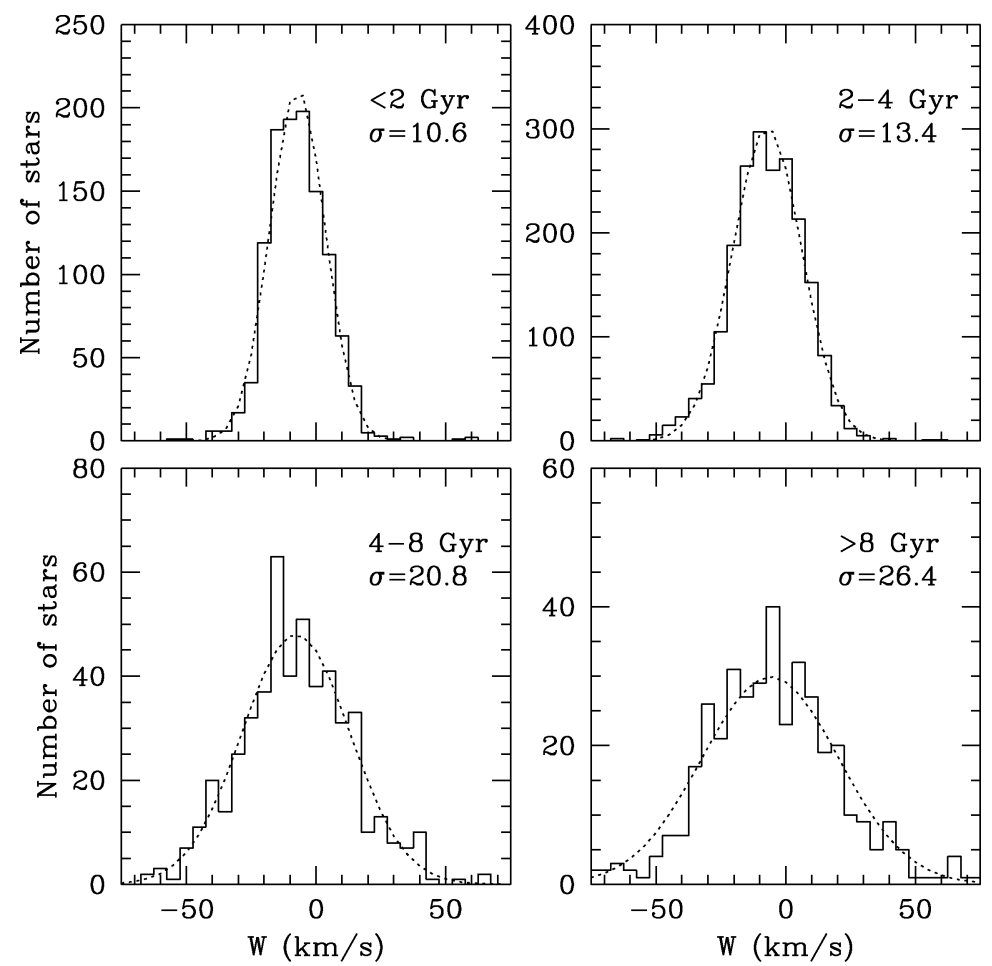

Figure 3. Distribution of $W$ velocities and fitted Gaussians for four age groups of the GCS (single stars with $\sigma($ Age $)<25 \%$; GCS II, Fig. 32). 
This does not, however, by itself mean that identifying the mechanism(s) responsible for the observed heating is a simple matter. Standard local gravity enhancement candidates are stationary spiral arms or density waves and giant molecular clouds (GMCs). However, such spiral arms do not affect the stellar motions in the vertical direction significantly, and GMCs are unable to reproduce the observed heating rate (Hänninen \& Flynn 2002).
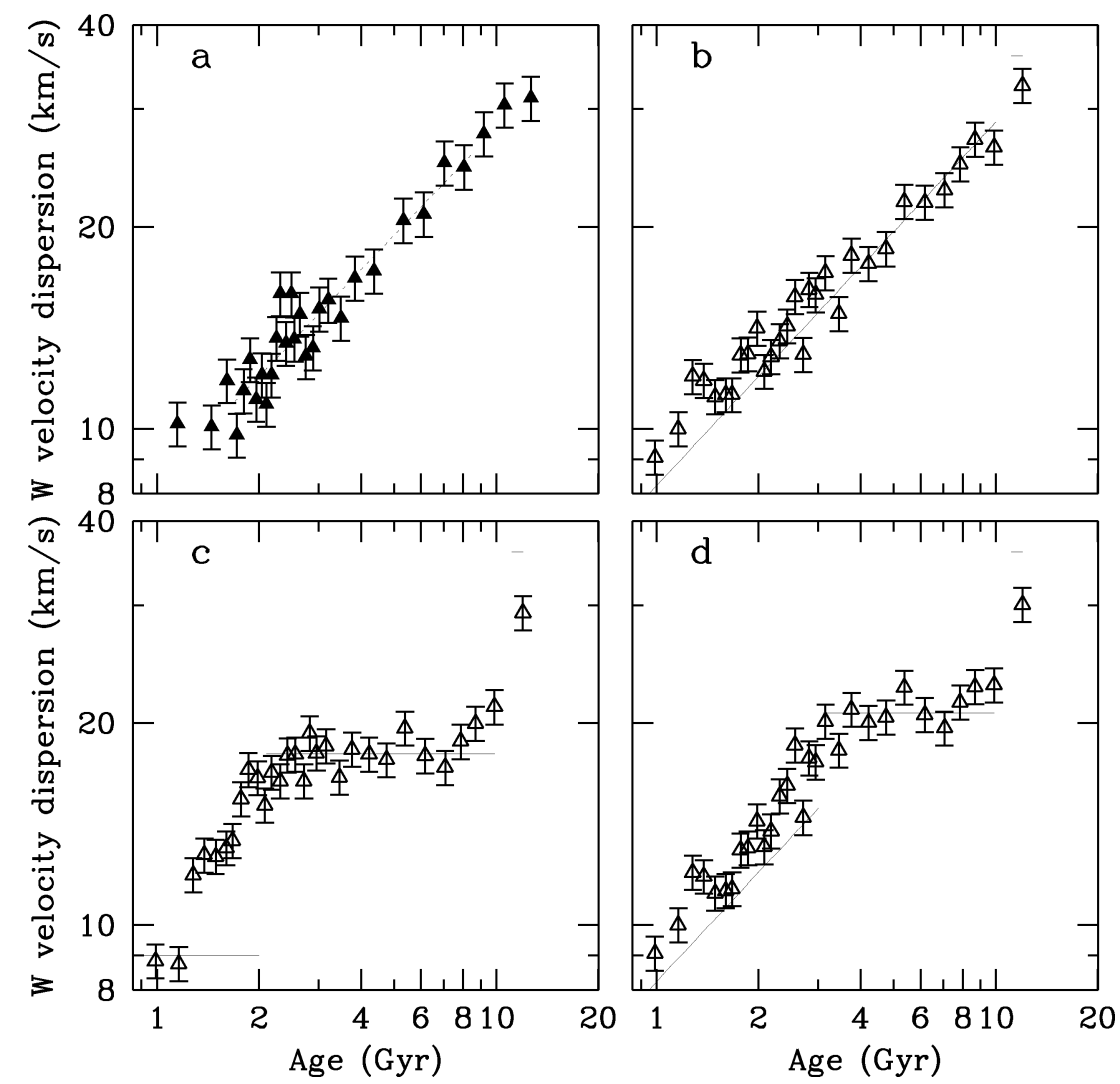

Figure 4. $a$ : Observed AVR in $W$ from GCS III (single stars with $\sigma_{A g e}<25 \%$ ) with a fitted power law. $b$-d: Simulated AVRs for three disk heating scenarios: Continuous heating, early saturation as in Quillen \& Garnett (2001), and a late minor merger. Open symbols in b, $c$, d: Rederived ages and velocity dispersions for the simulated stars.

Transient spiral arms appear to be able to match the data (De Simone et al. 2004), but their properties remain to be fully explored. More sophisticated calculations involve large-scale redistribution of stars within the disk over its lifetime without major net deformation of their initially circular orbits. Such models also seem able to reproduce the observed relations (see Roškar et al. 2008 and references therein), but offer few options for verification on kinematic grounds alone.

In the hierarchical merger scenario for galaxy formation, a galaxy like the Milky Way should be built up by gradual accretion of dwarf galaxies, such as the Sagittarius dwarf. Such mergers are expected to inject kinetic energy into the pre-existing (thin) disk stars, puffing up the disk and producing the observed heating. Satellites of appreciable mass are expected to produce a sharp jump in velocity dispersion or even disrupt the disk entirely; thus, the details of the $W$ velocity dispersion as a function of time - the agevelocity relation (AVR) - should provide significant clues to the actual history (see Fig. 4). 
For this to be possible, accurate stellar ages and reliable error estimates are obviously indispensable.

Fig. 4 suggests that the detailed shape of the AVR in $W$ should allow to discriminate between heating mechanisms internal to the disk and the effects of mergers. However, we note that the very recent calculations by Hopkins et al. (2008) indicate that, in more realistic merger scenarios, minor mergers would primarily heat the bulge of a spiral galaxy with little effect on the disk itself. If so, the two scenarios may indeed be indistinguishable from kinematic data alone, even with significantly larger samples and better ages.

\section{Conclusions}

Recent observational work has greatly improved the empirical data with which to test models of the dynamical heating of the disk. Further improvements in stellar age determination may allow us to refine the dynamical history of the disk in even greater detail than today, but this will require very significant improvements in the observational determination of effective temperatures and chemical compositions, and in the stellar models and their transformation to the observational plane. It is perhaps ironic that, at the same time, state-of-the-art dynamical simulations may be telling us that the ages and velocities of stars cannot tell us the full history of the dynamical history of the disk; very detailed chemical analyses ("chemical tagging") may be required as well.

Acknowledgements: Financial support from the Carlsberg Foundation, the Danish Natural Science Foundation and the International Astronomical Union is gratefully acknowledged. This research was also supported in part by the National Science Foundation under Grant No. PHY05-51164. I thank the Geneva-Copenhagen Survey Team for our pleasant collaboration on the whole project.

\section{References}

Bensby, T., Oey, M.S., Feltzing, S., \& Gustafsson, B. 2007, ApJ, 655, L89

Dehnen, W. 2000, AJ, 119, 800

Dehnen, W. \& Binney, J. J. 1998, MNRAS 298, 387

De Simone, R. S., Wu, X., \& Tremaine, S. 2004, MNRAS 350, 627

Famaey, B., Jorissen, A., Luri, X., et al. 2005, A\&A 430, 165

Holmberg, J., Nordström, B., \& Andersen, J. 2007, A\&A 475, 519 (GCS II)

Holmberg, J., Nordström, B., \& Andersen, J. 2009, AESA, in press (GCS III); also arXiv:0811.3982

Hopkins P. F., Hernquist, L., Cox, T. J., Younger, J. D., \& Besla, G. 2008, ApJ, in press; also arXiv:0806.2861v2

Hänninen, J. \& Flynn, C. 2002, MNRAS 337, 731

Jørgensen, B. R. \& Lindegren, L. 2005, A\&A 436, 127

Nordström, B., Mayor, M., Andersen, J., Holmberg, J., et al. 2004, A\&A 418, 989 (GCS I)

Quillen, A. C. \& Garnett, D. 2001, in Galaxy Disks and Disk Galaxies, eds. J. G. Funes, S. J. \& E. M. Corsini. ASP Conf. Ser. 230, 87

Roškar, R., Debattista, V. P., Quinn, T. R., Stinson, G. S., \& Wadsley, J. 2008, ApJ 684, L79

Seabroke, G. M. \& Gilmore, G. 2007, MNRAS 380, 1348

van Leeuwen, F. 2007, A\&SA 474, 653

Wielen, R. 1977, A\&A 60, 263

Zwitter, T. Siebert, A. Munari, U., et al. 2008, AJ 136, 421 


\section{Discussion}

J. Melbourne: I was again struck by the large scatter in metallicity at a range of ages from 5 Gyr on. Does this argue for a more extended formation age for the thick disk?

B. Nordström: The spread in metallicities is in fact large (and the same) for all ages. This could point to dynamical effects. New models by Roskar et al. (2008) and Schonrich \& Binney (2008) point to a kinematic origin of the spread.

I. KING: How good are your 3-D velocities? That is, how sure are you of assignment of each individual star to a particular moving group? In the past this was poor; studies of metallicities by Strömgren photometry in earlier days (e.g., Breger, PASP 1971) showed that only half the stars assigned to a group were really members.

B. Nordström: The 3-D velocities are accurate to $1.5 \mathrm{~km} \mathrm{~s}^{-1}$. This makes the verification of membership of the moving groups more certain than before. There might not be sharp borders to field stars. The groups seem to be an effect of dynamical mechanisms. The metallicities and ages of the "moving groups" do not point to a common birthplace.

D. Soderblom: You stated that studying a volume- limited sample of the solar neighborhood does not work for understanding some Galactic properties because models show the stars come from a large range of locations in the Galaxy. What would work?

B. Nordström: We should still look at a volume- limited sample and try to understand the large scatter in the diagnostic diagrams (age-metallicity and age-velocity). The scatter is much larger than the observational errors and I think that there is information there that tells us something. Many of the stars in the solar neighborhood follow the Sun, and others that are leaving might be replaced by similar stars. 


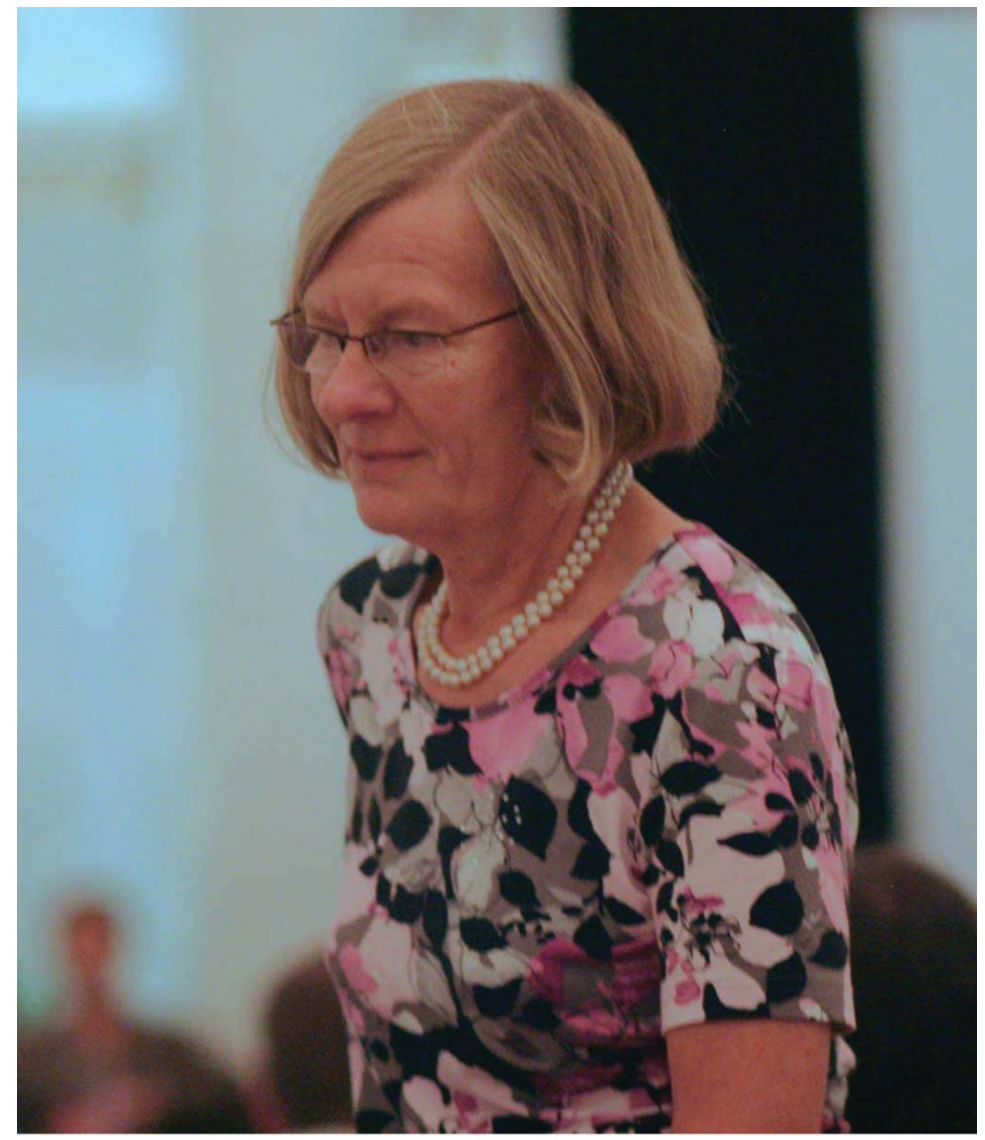

Birgitta Nordström 